\title{
SERVICE DIFFERENTIATION MECHANISM VIA COOPERATIVE MEDIUM ACCESS CONTROL PROTOCOL*
}

\author{
Fatma Orsun, Hakan Topakkaya, Muharrem A. Tunc and Coskun Cetinkaya \\ Department of Electrical and Computer Engineering \\ Wichita State University \\ \{fxorsun,hxtopakkaya,matunc, coskun.cetinkaya\}@wichita.edu
}

\begin{abstract}
Providing differentiated Quality of Service (QoS) levels is an important challenge for wireless ad hoc networks and wireless LANs when applications have diverse performance requirements. The IEEE 802.1 le MAC protocol can provide a Dynamic MAC by assigning different AIFSs, contention window expansion factors ( $\mathrm{PFs})$, and $\left(C W_{\min }, C W_{\max }\right)$ pairs for different classes and can provide a Static MAC by adjusting the durations of AIFSs based on priority levels [Aad01]. In this paper, we propose a novel and efficient service differentiation mechanism via the C-MAC. In our protocol, each node will change its backoff counter based on both its own packet's priority level and the priority level of the transmitted packet. The simulation results indicate that the Static MAC provides a service differentiation at the expense of significant goodput degradation when the amount of high priority class traffic is low. On the other hand, the Dynamic MAC fails to prevent low priority classes accessing the channel resulting in significant high priority class goodput degradation when the network load is high. However, our mechanism always provides an efficient service differentiation mechanism and high goodput with a small goodput degradation.
\end{abstract}

Keywords: Service Differentiation, MAC protocol

\section{Introduction}

Supporting differentiated Quality of Service (QoS) levels is an important challenge for wireless ad hoc networks and wireless LANs when applications have diverse performance requirements such as high goodput, low delay and delay jitter. The Medium Access Control (MAC) protocols must provide an effective mechanism to support the desired QoS levels while achieving high goodput and sharing limited spectrum resources fairly among the users.

\footnotetext{
*This material is based upon work supported by the National Science Foundation under Grant No. EPS0236913 and matching support from the State of Kansas through Kansas Technology Enterprize Corporation.
} 
The most popular wireless LAN, the IEEE 802.11 standard [802.11], has two different configurations: infrastructure and ad hoc modes. In the infrastructure mode, the IEEE 802.11 MAC can support the desired QoS levels via the Point Coordination Function (PCF). However, several researchers show that the PCF can result in poor performance under various scenarios [ Kuo97; San97]. In the ad hoc mode, a number of different MAC protocols have been proposed to provide service differentiation via a distributed reservationbased scheme or a contention-based scheme. The reservation-based scheme is explored to achieve service differentiation in [Lin99; Muir98; Sheu01]. Since these schemes require high power, memory, information exchanging, and computation complexity, they may not be suitable for some applications and environments where users have scarce resources. Further, when resources are reserved, but unused, they will be wasted, which will lead to low network utilization. In [Kon01], a packet's priority is piggybacked into the RTS/CTS/DATA/ACK frames, and each node maintains a scheduling table to provide dynamic priority. This scheme also requires high power, memory, information exchanging, and computation complexity. [Sob99; Yang02] provide service differentiation via "Black Burst" and "busy tone" which require jamming the channel before sending any data packets. However, these mechanisms can lead to significant goodput degradation under heavy network loads. It is also hard to implement these mechanisms using the IEEE 802.11 standard since they require significant changes. The IEEE 802.11e MAC protocol can provide a dynamic priority mechanism (Dynamic MAC) by assigning different Arbitration Inter Frame Spaces (AIFSs), contention window expansion factors (PFs), and $\left(C W_{\min }, C W_{\max }\right)$ pairs for different classes [802.11 e; Man02; Bar01; Zhu04; Ver01; Deng98; Rom03] and can provide a static priority mechanism (Static MAC) by adjusting the durations of AIFSs based on priority levels [Aad01].

In this paper, we propose a novel and efficient service differentiation mechanism via the Cooperative MAC protocol (C-MAC) [Cet04; CetTR], which is referred to as the SD-MAC. Our key technique is that each node will change its backoff counter based on both its own packet's priority level and the priority level of the transmitted packet. Specifically, a node will increase its backoff counter linearly with a higher priority (than its own) packet transmission and decrease it exponentially with a lower priority (than its own) packet transmission. We performed extensive sets of simulations in which we compared our mechanism with both the Static MAC and the Dynamic MAC in terms of the network goodput, the high priority class goodput, the packet loss rate, and admissible regions. The simulation results indicate that the Static MAC provides a service differentiation at the expense of significant goodput degradation when the amount of high priority class traffic is low. For example, when there is no high priority class traffic in the network, the Static MAC only achieves $27 \%$ goodput in the best case while the Dynamic MAC and the SD-MAC pro- 
vide high goodput, up to $71 \%$. On the other hand, the Dynamic MAC fails to prevent low priority classes accessing the channel when the network load is high. As a result, the high priority class traffic encounters a significant goodput degradation, specifically up to $25 \%$ less than the Static MAC and the SD-MAC, when the amount of low priority class traffic increases. Finally, we investigated the admissible regions, and the results show that the Dynamic MAC can not support any real time traffic when the non-real-time traffic is $70 \%$ or more of the channel data rate. On the other hand, the Static MAC encounters significant performance degradation when multiple real time traffic classes exist in the network. For example the Static MAC can support 2 voice and 12 VBR sources together while the SD-MAC supports 2 voice and 29 VBR or 12 VBR and 120 voice together. As a conclusion, the Static MAC is not an efficient mechanism to differentiate services with multiple real time classes, and the Dynamic MAC is not an efficient mechanism to protect real time traffic from greedy sources while the SD-MAC performs quite well under both scenarios. Hence, the SD-MAC is a good candidate for service differentiation.

The remainder of this paper is organized as follows. We propose a service differentiation mechanism, the SD-MAC, in Section 2, and describe the Static MAC and the Dynamic MAC in Section 3. Next, in Section 4, we present the comparison results of three mechanisms. Finally, we conclude in Section 5.

\section{Service Differentiation Mechanism}

In this section, we first briefly review the underlying MAC ptotocol, Cooperative MAC (C-MAC) [Cet04; CetTR]. Then, we describe the proposed service differentiation mechanism.

\section{Review of the Cooperative-MAC Protocol}

Unlike the IEEE 802.11 MAC protocol, in our protocol, we use a constant window size to reduce the amount of idle slots, and we resolve collisions among the collided nodes first by assigning these nodes a shorter DIFS than the other nodes, i.e. the other nodes have to wait for the channel to be idle for a longer duration of DIFS. The operation of the C-MAC protocol is as follows:

As in CSMA/CA, any active node senses the channel to send a packet. If it finds the channel to be idle for a duration of DIFS $(110 \mu \mathrm{sec})$, which is $50 \mu \mathrm{sec}$ in the IEEE 802.11 standard, the transmission will proceed. If the channel is determined to be busy, the node defers its transmission until the channel is idle for a duration of DIFS. Then, it generates a random backoff counter chosen uniformly from the range $[0, C W-1]$, where $\mathrm{CW}$ is the contention window size. The backoff counter is decremented as long as the channel remains idle. If it becomes busy, the counter is frozen until the channel is again sensed to be idle for a duration of DIFS. 
When the backoff counter reaches 0 , the node transmits an RTS message if the four-way hand-shake mechanism is used. The receiving node responses with a CTS after a time period of SIF $(10 \mu \mathrm{sec})$. Any other nodes which hear RTS/CTS packets defer their transmissions and update their NAV. The sender node responses to the CTS with an actual data packet and waits for an ACK packet. If a node has a successful transmission, it chooses a new backoff counter uniformly from the range $[C W, 2 \cdot C W-1]$ in order to let the deferred nodes capture the channel. This will lead to short-term fairness. If the sender node does not hear a CTS/ACK, it assumes that a collision has occurred and proceeds according to the procedure described below.

If a node encounters a collision, it generates a new backoff counter chosen uniformly from the range $[0,3]$ and starts decrementing the counter if it finds the channel to be idle for a duration of Priority Inter-Frame Space (PIFS, $30 \mu \mathrm{sec})$ rather than a duration of DIFS. The DIFS $(110 \mu \mathrm{sec})$ corresponds to

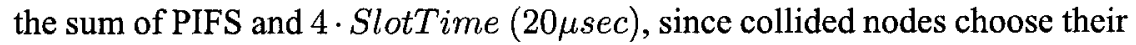
backoff counters from the range [0,3]. Therefore, the collided nodes have a higher priority than the other nodes in terms of packet transmission. If a collided node senses another collision in the channel before its counter reaches 0 , it sets its counter to 0 and waits for the channel to be idle for a duration of DIFS to transmit. As a result, it has a higher priority than the nodes which are not involved in a collision. Any node that does not receive an RTS/CTS correctly is able to sense that the medium is busy since the interference power received is sufficiently higher than the noise floor. Therefore, it assumes that a collision occurred, and it sets its NAV to the Extended Interframe Space (EIFS) [802.11; Kwon03]. After a successful transmission, the node chooses a new backoff counter uniformly from the range $[C W, 2 \cdot C W-1]$ and needs to detect a DIFS amount of idle time to reduce its backoff counter. (Please see our technical report for more details [CetTR]).

\section{Service Differentiation Mechanism}

In this section, we describe our service differentiation mechanism, SDMAC, via the cooperative MAC protocol (C-MAC) presented above for wireless ad hoc networks and wireless LANs. Our key technique is that each node will change its backoff counter based on both its own packet's priority level and the level of the transmitted packet. Specifically, a node will increase its backoff counter linearly with a higher priority (than its own) packet transmission and decrease it exponentially with a lower priority (than its own) packet transmission. From now on, we assume that each node uses static priority among its packets to schedule the next packet transmission.

The SD-MAC first assigns different PIFSs and DIFSs values for different classes to provide service differentiation. We set the $P I F S^{i}=P I F S^{i-1}+$ st and $D I F S^{i}=D I F S^{i-1}+s t$ in order to provide high network goodput under a low amount of high priority class traffic. Second, the SD-MAC chooses a 
backoff counter from the range $[0,3]$ after a collision as in the C-MAC. However, a node with a priority level $i$ packet to transmit will always choose its backoff counter from the range $\left[C W^{i}, 2 \cdot C W^{i}-1\right]$ where $C W^{i+1} \geq 2 \cdot C W^{i}$. Therefore, the high priority classes will capture the channel initially. However, after some time period, low priority classes will decrement their backoff counter and start competing with high priority classes. As a result, the high priority classes would encounter significant goodput degradation similar to what occurs in the Dynamic MAC (Please see Figures 1).

In order to prevent this phenomenon, the SD-MAC requires the low priority classes to increase their backoff counters by one (1) when they hear a higher priority (than its own) packet transmission. However, we should limit the increment such that low priority classes should quickly capture the channel after a burst of high priority traffic in order to increase the network goodput. Therefore, the node will not increase its backoff counter more than $2 \cdot C W^{i}$. This method increases the chance of a high priority class packet capturing the channel while it assures that the backoff counter of low priority class does not exceed the maximum possible value, and it is very efficient under a heavy load of high priority classes. In addition, low priority classes still can capture the channel especially when a low amount of high priority traffic is present. However, to increase high priority class goodput, the high priority classes should capture the channel quickly if they hear any low priority class transmissions. To achieve this goal, upon hearing a lower priority (than its own) packet transmission, a node waits for the channel to be idle for $P I F S^{i}$ amount of time, instead of $D I F S^{i}$. Then, it reduces its backoff counter to half (exponential decrease) for each detected idle slot. This procedure enables high priority classes to capture the channel in a very short amount of time. If a collision is sensed during this procedure, the exponential decrement will be stopped and the regular linear decrement will be activated, i.e. the node will wait for the channel to be idle for a duration of DIF $S^{i}$ amount and decrement its backoff counter by one (1) for every detected idle slot.

The simulation results show that the SD-MAC still provides short-term fairness (2-3 packets per user) within the classes since it is based on the C-MAC protocol, and the proposed mechanism generates cohesive group movement although each user acts independently. (We omit the figures because of space limitation). However, the SD-MAC requires an additional look up into the packet header in order to identify the priority level of the transmitted packet. In simulations, we set the $P I F S^{1}=30 \mu s e c, D I F S^{1}=110 \mu s e c, P I F S^{2}=$ $50 \mu \mathrm{sec}$, and $D I F S^{2}=130 \mu \mathrm{sec}$. We use sets of $\left(C W^{1}, C W^{2}\right)=\{(4,8),(8,16)$, $(16,32),(32,64)\}$. We also set $C W^{1}$ to a fix number and vary the $C W^{2}$ up to 128 . We found that the $C W^{2}>2 \cdot C W^{1}$ results in either the same as $C W^{2}=2 \cdot C W^{1}$ or a negligible performance improvement. 


\section{Reviews of the Static MAC and the Dynamic MAC Protocols}

\section{The Static MAC Protocol}

The Static MAC protocol, introduced in [Aad01], employs the IEEE 802.11 DCF as an underlying access method. It uses different AIFS values, where AIFS corresponds to the DIFS in the regular IEEE 802.11 MAC, for different priority levels. The AIFS of the level $(i+1)$ is set to the sum of the AIFS of level $i$ and the time duration of the maximum contention window size of level $i\left[\left(C W_{\max }^{i}+1\right) * s t\right]$ where $s t$ is the slot time. For example, in the case of two priority classes, let $A I F S^{1}$ be equal to $30 \mu s e c, C W_{\text {max }}^{1}$ equal to 63 , and $s t$ equal to $20 \mu \mathrm{sec}$. Then, $A I F S^{2}=A I F S^{1}+\left(C W_{\max }^{1}+1\right) * s t$ which is equal to $1.31 \mathrm{msec}$. In our simulations, we set $A I F S^{1}=30 \mu \mathrm{sec}, C W_{\min }^{1}=15$, $C W_{\min }^{2}=31$, and $C W_{\max }^{2}=1023$. We vary the $C W_{\max }^{1}$ from 63 to 511 , then we use the above equation to calculate $A I F S^{2}$.

\section{The Dynamic MAC Protocol}

The IEEE 802.11e [802.11e] is the de facto standard for Quality of Service provisioning. The IEEE $802.11 \mathrm{e}$ uses a dynamic priority mechanism by assigning different AIFS values, contention window expansion factors ( $\mathrm{PFs}$ ), and $\left(C W_{\min }, C W_{\max }\right)$ pairs for different classes to provide priority among them. The IEEE 802.11e, which is referred to as the Dynamic MAC, employs DCF as an underlying access method.

The first component is the required idle time to access the channel, i.e., AIFS. In this mechanism, the highest priority class has the shortest AIFS while the lowest class has the longest. $A I F S^{i}$ is calculated as: $A I F S^{i}=$ $A I F S^{i-1}+$ st. The second component is the backoff procedure. The basic IEEE 802.11 access mechanism always uses an exponential factor of 2 . On the other hand, the Dynamic MAC provides different exponential increase factors (PFs) which increase with decreasing priority levels. Further, each class has different values of $C W_{\min }$ and $C W_{\max }$. As a result of these settings, a high priority class waits a smaller duration to reduce its backoff counter, chooses its backoff counter from the smaller value range, and increases its $\mathrm{CW}$ value slower than a lower priority class. However, if high priority class has a higher density than low priority classes, it may encounter a high collision rate. Therefore, it may have a higher $\mathrm{CW}$ value and loss its priority. In simulations, we set $A I F S=\{30 \mu \mathrm{sec}, 50 \mu \mathrm{sec}\}, P F=\{2,4\}, C W_{\min }=\{15,31\}$, and $C W_{\max }^{2}=1023$, and we vary $C W_{\max }^{1}$ from 63 to 511 .

\section{Comparison of the Service Differentiation Mechanisms}

In this section, we describe the simulation model, then we compare the SDMAC with the Static MAC and Dynamic MAC in terms of network goodput, Class 1 goodput, and admissible regions. 


\section{Simulation Model}

We use the ns-2 simulator NS-2 with the DSSS specification and $11 \mathrm{Mbps}$ Data Rate. We have three traffic models which are used in [Sheu01]. The Voice Traffic generates a $64 \mathrm{Kbps}$ constant bit rate in the on state and no traffic in the off state. We also model both the on and off states as exponential distributions with the mean duration of 1 and 1.35 seconds respectively. Additionally, the maximum delay bound for voice traffic is set to $25 \mathrm{msec}$. The VBR Traffic generates $120 \mathrm{Kbps}$ minimum rate, $240 \mathrm{Kbps}$ average rate, and $420 \mathrm{Kbps}$ maximum rate. The maximum delay bound for video traffic is set to $75 \mathrm{msec}$. The Data Load for a single source is modelled as a constant bit rate traffic with a rate of $1 \%$ of the channel data rate.

We use two performance metrics to evaluate service differentiation mechanisms: the Goodput and the Packet Loss Rate (PLR). The Goodput is defined as the amount of the applications' data transferred over the wireless link and normalized by the channel data rate. If a packet can not be delivered within the delay bound, it is dropped. The Packet Loss Rate (PLR) is defined as the ratio of dropped packets due to a delay bound violation over the total number of packets generated by real-time traffic. We use two scenarios: (1) voice traffic as Class 1 (high priority class) traffic and (2) VBR tarffic as Class 1. Data load is set to Class 2 (low priority class) traffic under both scenarios and is varied from 0 to 1.5 via adding more sources. Also, the number of voice traffic sources is varied from 0 to 300 and VBR traffic from 0 to 40 .

\section{Performance Comparison}

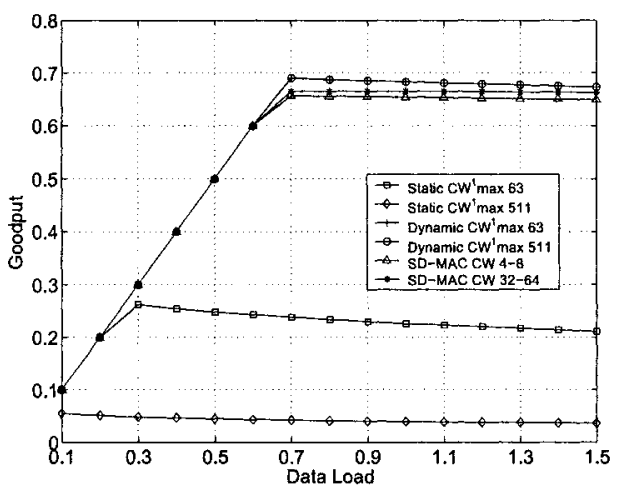

(a) No Class 1 Traffic

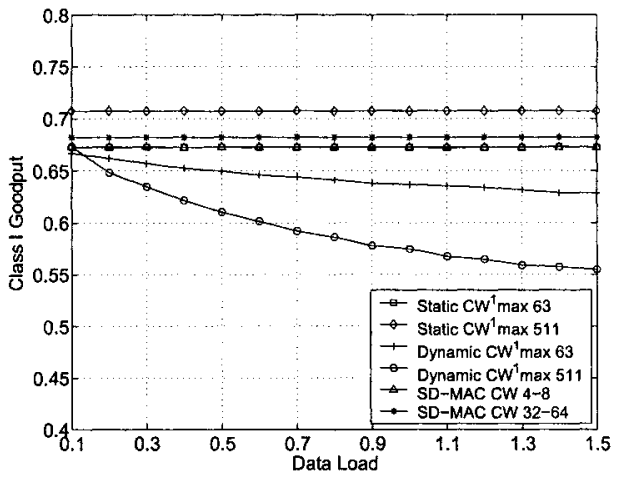

(b) The Class 1 Goodput for 40 VBR

Figure 1. The Goodput Comparison

First, we investigate the case in which no Class 1 traffic is present. As shown in the Figure 1 (a), the SD-MAC and the Dynamic MAC provide high network goodput (up to $67 \%$ and $70 \%$ respectively) while the Static MAC encounters 
significant goodput degradation ( $27 \%$ maximum). Therefore, the Static MAC may not be a good mechanism for service differentiation. Next, we consider a heavy real-time traffic load case. We depicted the Class 1 goodput for 40 VBR sources in Figures 1 (b). When the Class 1 goodput is considered, the Static MAC and the SD-MAC provide steady goodput, i.e. they are able to protect the Class 1 traffic from Class 2 traffic efficiently. However, the Dynamic MAC fails to prevent low priority classes accessing the channel. As a result, the Dynamic MAC provides up to $22 \%$ and $25 \%$ less Class 1 goodput for voice and VBR traffics respectively when compared with the SD-MAC. As a conclusion, the Dynamic MAC may not be a good mechanism for service differentiation.

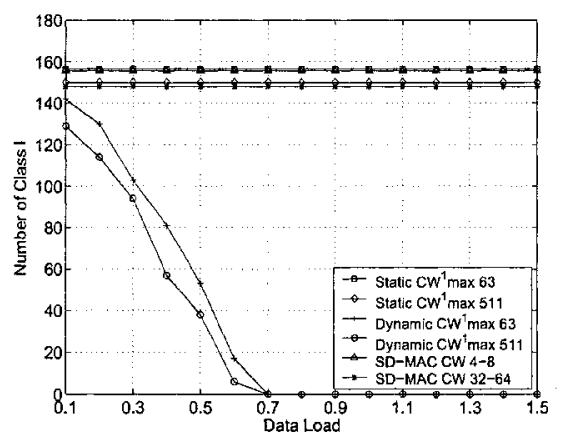

(a) Voice Traffic

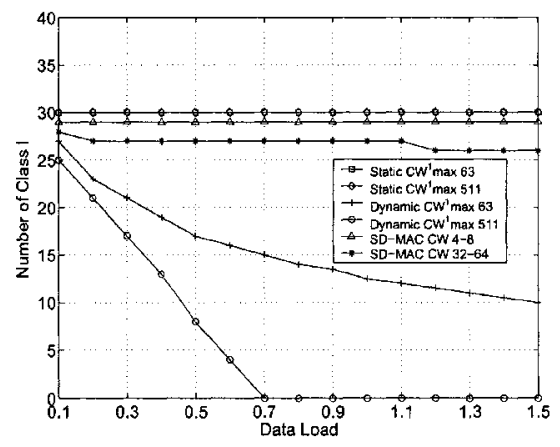

(b) VBR Traffic

Figure 2. The Admissible Region for Voice and VBR Traffic under Data Load

Next, we investigate the admissible regions, the number of users to be admitted to the network with a delay guarantee when the real-time traffic requires a maximum of $\left(10^{-3}\right)$ packet loss rate. The first scenario sets real-time traffic as Class 1 traffic and data load as Class 2 traffic. We depicted the admissible regions for the voice traffic and VBR traffic in Figures 2 (a) and (b) respectively. The Static MAC yields the largest admissible region and supports up to $5 \%$ more real-time traffic than the SD-MAC. However, for the CW pair is $(16,32)$ or lower values, the difference is reduced to $1 \%$. On the other hand, the Dynamic MAC yields the smallest admissible region. For example, even in the presence of a $10 \%$ data load, the Dynamic MAC supports up to $21 \%$ less real-time traffic. Further, it can not support any real-time traffic if the data load is $70 \%$ or more of the channel data rate in the most cases.

In the second scenario, we choose voice traffic as Class 1 traffic and VBR traffic as Class 2 traffic. We depicted the admissible region in Figure 3. The $\mathrm{SD}-\mathrm{MAC}$ with $\mathrm{CW}=(4,8)$ yields the largest admissible region, and the Dynamic MAC performs very close to the SD-MAC while the Static MAC pro- 


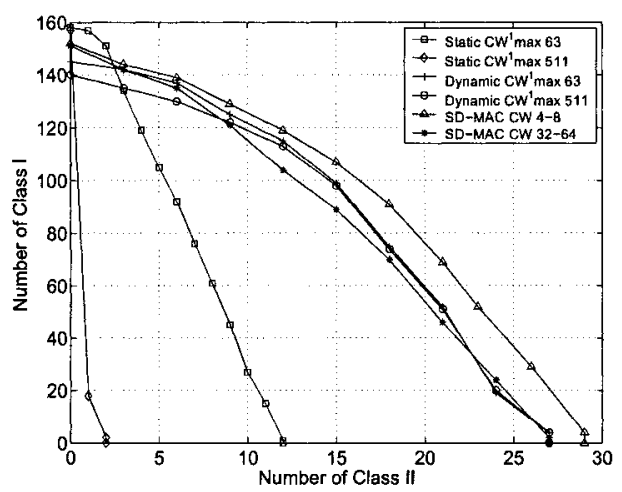

Figure 3. The Admissible Region for Voice Traffic vs. VBR Traffic

vides significantly a smaller admissible region. For example, the Static MAC can support 2 voice and 12 VBR sources together while the SD-MAC can support 2 voice and 29 VBR sources or 12 VBR and 120 voice sources. As a conclusion, the Static MAC is not an efficient mechanism to differentiate among multiple real-time traffic classes, and the Dynamic MAC fails to protect the real-time traffic from greedy sources. However, the SD-MAC performs quite well under the both scenarios. Therefore, the SD-MAC is a good candidate for service differentiation.

\section{Conclusions}

The goal of this work is to design a distributed medium access protocol to provide service differentiation without sacrificing the goodput, delay, and fairness when applications have diverse performance requirements. Towards this end, we propose a novel and efficient differentiated service mechanism via the C-MAC for wireless ad hoc networks and wireless LANs. Our key technique is that each node will change its backoff counter based on both its own packet's priority level and the priority level of the transmitted packet.

We compared our mechanism with both the Static MAC and the Dynamic MAC via simulations. The simulation results indicate that the Static MAC provides a service differentiation at the expense of significant goodput degradation when the amount of high priority class traffic is low. On the other hand, the Dynamic MAC fails to prevent low priority classes accessing the channel when the network load is high. Further, the Static MAC is not an efficient mechanism to differentiate among multiple real-time traffic classes, and the Dynamic MAC is not an efficient mechanism to protect real time traffic from greedy sources. However, our mechanism always provides an efficient service differentiation mechanism and high goodput with a small goodput degradation. 


\section{References}

IEEE 802.11e. Medium Access Control (MAC) and Physical Layer (PHY) Specifications: MAC Enhancements for Quality of Service (QoS). May 2002.

I. Aad and C. Castelluccia. Differentiation mechanisms for IEEE 802.11. In Proceedings of IEEE INFOCOM 2001, Anchorage, Alaska, Apr. 2001.

C. Cetinkaya and F. Orsun. Cooperative Medium Access Control for Dense Wireless NetworksTechnical Report. http://www.engr.wichita.edu/esawan/TC-Cetinkaya-CMAC.pdf.

J. Sanchez, R. Martinez, and M.W. Marcellin. A Survey of MAC Protocols Proposed for Wireless ATM. IEEE Networks, vol. 11, no. 6, pp. 52-62, Nov. 1997.

W.K. Kuo, C.Y. Chan, and K.C. Chen. Time bounded Services and Mobility Management in IEEE 802.11 Wireless LANs. In Proceedings of IEEE Personal Wireless Comm., 1997.

C. Cetinkaya and F. Orsun. Cooperative Medium Access Control for Dense Wireless Networks. In Proceedings of Med-HOC 2004, Bodrum, Turkey, June 2004.

Y. Kwon, Y. Fang, and H. Latchman. A Novel MAC Protocol with Fast Collision Resolution for Wireless LANs. In Proceedings of IEEE INFOCOM 2003, San Francisco, CA, Apr. 2003.

VINT group. UCB/LBNL/VINT Network Simulator-ns (Version 2). http://mash.cs.berkeley.edu/ns.

IEEE. IEEE Standard 802.11: Wireless LAN Medium Access Control (MAC) and Physical Layer (PHY) Specifications. 1997

V. Kanodia, C. Li, A. Sabharwal, B. Sadeghi, and E. Knightly. Distributed Multi-hop Scheduling and Medium Access with Delay and Throughput Constraints. In Proceedings of $A C M$ MOBICOM '01, Rome, Italy, July 2001.

S. Mangold et al. The IEEE 802.11e MAC for Quality of Service in Wireless LANs. In Proceedings of European Wireless, Feb. 2002.

X. Yang and N. H. Vaidya. Priority scheduling in wireless ad hoc networks. In Proceedings of ACM MobiHoc '02, Lausanne, Switzerland, July 2002.

J. L. Sobrinho and A. S. Krishnakumar. Quality-of-Service in Ad Hoc Carrier Sense Multiple Access Wireless Networks. IEEE Journal on Selected Areas in Communications, vol. 17, Aug. 1999.

M. Barry, A. T. Campbell, and A. Veres. Distributed Control Algorithms for Service Differentiation in Wireless Packet Networks. In Proceedings of IEEE INFOCOM 2001, Anchorage, Alaska, Apr. 2001.

H. Zhu, G. Cao, A. Yener, and A. D. Mathias. EDCF-DM: A Novel Enhanced Distributed Coordination Function for Wireless Ad Hoc Networks. In Proceedings of IEEE ICCC'04, Paris, France, June 2003.

A. Veres et al. EDCF-DM: Supporting service differentiation in wireless access packet networks using distributed control. JSAC, vol. 19, no. 10, Oct. 2001.

S. T. Sheu and T. F. Sheu. DBASE: A Distributed Bandwidth Allocation/Sharing/Extension Protocol for Multimedia over IEEE 802.11 Ad Hoc Wireless LAN. In Proceedings of IEEE INFOCOM 2001, Anchorage, Alaska, Apr. 2001.

L. Romdhani, Q. Ni, and T. Turletti. Adaptive EDCF: Enhanced Service Differentiation for IEEE 802.11 Wireless Ad Hoc Networks. In Proceedings of IEEE WCNC'03, 2003.

J. Deng and R. S. Chang. A priority Scheme for IEEE 802.11 DCF Access Method. IEICE Transactions in Communications, vol. 82-B, no. 1, Jan. 1999.

A. Muir and J. J. Garcia-Luna-Aceves. An Efficient Packet Sensing MAC Protocol for Wireless Networks. ACM Journal on Mobile Networks and Applications, vol. 3, no. 2, Aug. 1998.

C. R. Lin and M. Gerla. Real-time support in multihop wireless networks. Wireless Networks, vol. 5, 1999. 